\title{
Evaluation of treatment outcomes of intravenous ceftazidime as the intensive phase antimicrobial in melioidosis - a case series.
}

\author{
AN KuruppuArachchi ${ }^{1}$, VP Jayasinghe ${ }^{1}$, KL Thebuwana ${ }^{1}$, D Jayawardane ${ }^{2}$, KT Sundaresan ${ }^{2}$, M \\ Umakanth $^{2}$, P Mayurathan ${ }^{2}, \mathrm{~K}$ Thambiretnnam ${ }^{2}$
}

\section{Introduction}

Melioidosis is an infection caused by the Gram negative bacterium, Burkholderiapseudomallei. It is associated with high morbidity and mortality even after treatment with potent antibiotics. We present four cases of culture positive melioidosis, treated with intravenous ceftazidime as the antimicrobial agent in the intensive phase. Our main objectives were to analyze morbidity, mortality and time taken for clinical remission following intensive phase treatment with intravenous ceftazidime.

\section{Methods}

This is a retrospective study carried out over a period of 6 months from January 2017 at the Teaching Hospital Batticaloa, Sri Lanka. All culture proven cases of melioidosis admitted to medical and surgical wards were included. Intravenous ceftazidime for 28 days, with or without co-trimoxazole, was given to all patients during the intensive phase. Absence of fever and a reduction of the CRP by $50 \%$ of its highest value were taken as indicative of clinical remission.

\section{Results}

There were four culture positive patients of whom three had positive blood cultures and one had a positive sputum culture. They showed nonspecific clinical presentations such as skin abscesses, splenic abscesses, lung abscesses and septic arthritis. All patients had near total recovery after intensive phase of chemotherapy for 28 days and there were no deaths compared with the high mortality rate in disease endemic areas of the world. This could be due to early clinical detection and appropriate and long course of antibiotics. Mean fever clearance time was 8.33 days. Mean time taken for highest CRP value to reduce by $50 \%$ was 6.66 days. Early clinical remission in patient with septic arthritis may be due to arthrotomy and washout

\section{Conclusion}

The satisfactory outcome of these cases highlights the importance of early diagnosis with a high index of suspicion, correct use of antibiotics and improved microbiology services. However, adequate sample size is needed to determine the clinical significance of these findings.

\footnotetext{
${ }^{1}$ Department of Medicine, Teaching Hospital, Batticaloa, Sri Lanka

${ }^{2}$ Department of Microbiology, Teaching Hospital, Batticaloa, Sri Lanka

${ }^{3}$ Department of Surgery, Teaching Hospital Batticaloa, Sri Lanka

Address for correspondence: Dr DushaniJayawardane, Department of Microbiology, Teaching Hospital, Batticaloa, Sri Lanka.+94 777536038 Email: dushanidj@yahoo.com 1 https://orcid.org/0000-0003-2369-4976
} 